\title{
Feedback-Assisted Iterative Learning Model Predictive Control with Nonlinear Fuzzy Model
}

\author{
Xiangjie Liu and Ke Xi \\ State Key Laboratory of Alternate Electrical Power System with Renewable Energy Sources, North China Electric Power University, \\ Beijing 102206, China
}

Correspondence should be addressed to Xiangjie Liu; liuxj@ncepu.edu.cn

Received 28 February 2014; Revised 20 April 2014; Accepted 27 April 2014; Published 12 June 2014

Academic Editor: Changzhi Wu

Copyright (C) 2014 X. Liu and K. Xi. This is an open access article distributed under the Creative Commons Attribution License, which permits unrestricted use, distribution, and reproduction in any medium, provided the original work is properly cited.

Iterative learning control (ILC), due to its advantage of requiring less system knowledge, can serve as a feedforward signal in system control. ILC can be combined with model predictive control (MPC) to constitute a feedforward-feedback configuration. In this scheme, ILC provides most of the control signal and copes with the repetitive disturbances. MPC provides the supplementary control for regulation purpose and also for nonrepeating disturbance rejection. Considering the nonlinear industrial process, this paper establishes the plant nonlinear fuzzy model to constitute the fuzzy model-based feedback-assisted ILC. The integrated control strategy can achieve wide-range operation and good tracking performance. The performance of the feedback-assisted ILC is illustrated by a steam-boiler system.

\section{Introduction}

Ever since the original work on iterative learning control (ILC) [1], it has been researched and applied widely in industrial robotics $[2,3]$ and manufacturing process $[4]$ as a learning-type strategy. The objective of ILC is to use information obtained from past iteration runs to track a predefined reference and reject repetitive disturbances iteratively. The major advantage of ILC over traditional control algorithms is that it requires less computational efforts and less exact knowledge of the system. Thus it has drawn extensive attention in recent years [5-7].

However, ILC can be considered as an open-loop control scheme that is only capable of rejecting repetitive disturbances and cannot deal with nonrepeating disturbances efficiently. For this, ILC is often combined with a well-designed feedback controller. In this scheme, the tracking performance is guaranteed by ILC, which acts as a feedforward signal and provides most of control signals. The conventional feedback control path is meant to be supplementary to guarantee the system robustness to noises and disturbances.

The quadratically optimal ILC (Q-ILC) [8] was early constituted to be a model-based ILC, since it adopted a quadratic performance criterion to obtain an optimal learning gain. Its property provides a chance to incorporate feedback model predictive control (MPC) into ILC in order to improve the capability of rejecting disturbances.

Recently, various advanced MPC-ILC schemes have been proposed. Lee et al. [9] proposed the batch MPC (BMPC) to incorporate real-time feedback into Q-ILC to handle nonrepeating disturbances. By combining and modifying the existing Q-ILC and BMPC, Chin et al. [10] proposed a two-stage ILC framework to ensure that ILC control was unaffected by real-time disturbances.

In existing nonlinear MPC-ILC schemes, the plant model was usually expanded in Taylor's series, which was only valid in a normal operation point. Since the nonlinear industry process dynamics may vary in a large operating range, the model errors would inevitably introduce additional perturbations and deteriorate the control effect.

One way to deal with such difficulty is to develop the high accuracy plant model. In this paper, the Takagi-Sugeno (T-S) fuzzy model [11] is utilized to approximate the nonlinear system. The T-S fuzzy model, adopting the experts' experience, has the characteristic of simple structure and strong approximation ability. It is composed of a number of local 
linear models. The parameters of each local model are passed through the membership functions to generate an overall nonlinear model. Thus the proposed controller is a plantwide controller rather than a linear controller. With higher model exactness, the iteration process can converge to the predefined trajectory more quickly and tracking performance can also be improved.

In power generation industrial process, the steam-boiler generations in power plant should meet the load demand of power system under wide-range operation. The processes are complex systems with strong nonlinearity and subject to external disturbances. Since the dynamic of the steam-boiler system in power plant can be depicted as load dependent, T-S fuzzy model was established to represent the system dynamic. Thus the feedforward- (FF-) feedback (FB) control strategy is particularly suitable for controlling the system [12].

The aim of this paper is to derive a nonlinear FF-FB ILC based on fuzzy modeling technique. Section 2 describes the overall FF-FB control scheme. Section 3 presents the feedforward ILC control strategy. Section 4 incorporates MPC into ILC to formulate the FF-FB scheme. A simulation on the steam-boiler system is presented in Section 5 to illustrate the performance of the proposed feedback-assisted ILC. Finally, the conclusion is drawn in Section 6.

\section{The Overall Control Law}

Generally, the FF-FB strategy in a repetitive system can be expressed as

$$
u_{k}(t)=u_{k}^{\mathrm{ff}}(t)+u_{k}^{\mathrm{fb}}(t)
$$

where $k$ denotes the iteration number and $t$ denotes the current time, $u_{k}^{\mathrm{ff}}(t)$ represents the feedforward control signal in the $k$ th iteration at time $t, u_{k}^{\mathrm{fb}}(t)$ represents the feedback control signal in the $k$ th iteration at time $t$, and $u_{k}(t)$ is the overall control signal that will be applied to the process. The FF-FB controller structure is illustrated in Figure 1.

In Figure $1, u_{k}^{\mathrm{ff}}(t)$ is the feedforward portion which is provided by ILC updating law. $u_{k}^{\mathrm{fb}}(t)$ is the feedback portion which is a nonlinear MPC control signal. $u_{k}(t)$ is the total control signal in the $k$ th iteration. $y_{d}$ is the desired output reference, $e_{k}(t)$ is the error in the $k$ th iteration, and $y_{k}$ is the plant output in the $k$ th iteration.

\section{The ILC Control Law}

A nonlinear multi-input multi-output (MIMO) discrete-time system is written in the form of

$$
y=f(u, d)
$$

where $f$ denotes the nonlinear relationship between the input $u$, the disturbances $d$, and the output $y$.
Define the following sequences $u, y$, and $d$ as

$$
\begin{gathered}
\left.u \stackrel{\Delta}{=} \begin{array}{llll}
u^{T}(0) & u^{T}(1) & \cdots & u^{T}(N-1)
\end{array}\right]^{T} \in R^{n_{u} N}, \\
y \stackrel{\Delta}{=}\left[\begin{array}{lllll}
y^{T}(1) & y^{T}(2) & \cdots & y^{T}(N)
\end{array}\right]^{T} \in R^{n_{y} N}, \\
d \stackrel{\Delta}{=}\left[\begin{array}{lllll}
d^{T}(1) & d^{T}(2) & \cdots & d^{T}(N)
\end{array}\right]^{T} \in R^{n_{y} N},
\end{gathered}
$$

where the run length is fixed with $N$ sample steps and the dimensions of input and output are $n_{u}$ and $n_{y}$, respectively.

The nonlinear mapping $f$ can be presented by the T-S fuzzy model:

$$
\begin{aligned}
& R^{i}: \text { If } z_{1} \text { is } A_{i 1} \text { and } \cdots \text { and } z_{p} \text { is } A_{i p}, \\
& \text { then } y^{i}=G^{i} u-p^{i}, \quad i=1,2, \ldots, r
\end{aligned}
$$

where $r$ is the number of the rules, $R^{i}$ denotes the $i$ th rule, $z_{1} \cdots z_{p}$ are variables of the premise of implication, $A_{i 1} \cdots A_{i p}$ are fuzzy sets in the premise, $p^{i}$ denotes the total effects of disturbances and bias errors in the $i$ th subarea, and $G^{i}$ is impulse response coefficient matrix of the $i$ th submodel.

The FF-FB scheme can be written as

$$
u=u^{\mathrm{ff}}+u^{\mathrm{fb}}
$$

Combining (4) and (5), the input-output relationship can be rewritten as

$$
\begin{aligned}
& R^{i}: \text { If } z_{1} \text { is } A_{i 1} \text { and } \cdots \text { and } z_{p} \text { is } A_{i p}, \\
& \quad \text { then } y^{i}=G^{i}\left(u^{\mathrm{ff}}+u^{\mathrm{fb}}\right)-p^{i}, \quad i=1,2, \ldots, r .
\end{aligned}
$$

Then $y^{i}$ can be decomposed as $\bar{y}^{i}$ and $\widehat{y}^{i}$. Consider

$$
\begin{gathered}
\bar{y}^{i}=G^{i} u^{\mathrm{ff}}-p^{i}, \\
\widehat{y}^{i}=G^{i} u^{\mathrm{fb}}=G^{i} \widehat{u},
\end{gathered}
$$

where $\bar{y}^{i}$ is the suboutput of feedforward ILC control law in the $i$ th subarea and $\widehat{y}^{i}$ is the suboutput of feedback MPC control law in the $i$ th subarea. $\widehat{u}$ denotes $u^{\mathrm{fb}}$.

$G^{i}$ has the following lower-block triangular structure:

$$
G^{i}=\left[\begin{array}{cccc}
g_{1,0}^{i} & 0 & \cdots & 0 \\
g_{2,0}^{i} & g_{2,1}^{i} & \cdots & 0 \\
\vdots & \vdots & \ddots & 0 \\
g_{N, 0}^{i} & \cdots & \cdots & g_{N, N-1}^{i}
\end{array}\right] \in R^{n_{y} N \times n_{u} N}
$$

where $g_{p q}^{i} \in R^{n_{y} \times n_{u}}$ is the impulse response coefficient matrix which is attained by applying unit pulse inputs at time $q$ to outputs at time $p$ independently.

The integral system output $\bar{y}$ can be formulated in the following form:

$$
\bar{y}=\frac{\sum_{i=1}^{r} w_{i}(z) \bar{y}^{i}}{\sum_{i=1}^{r} w_{i}(z)}
$$




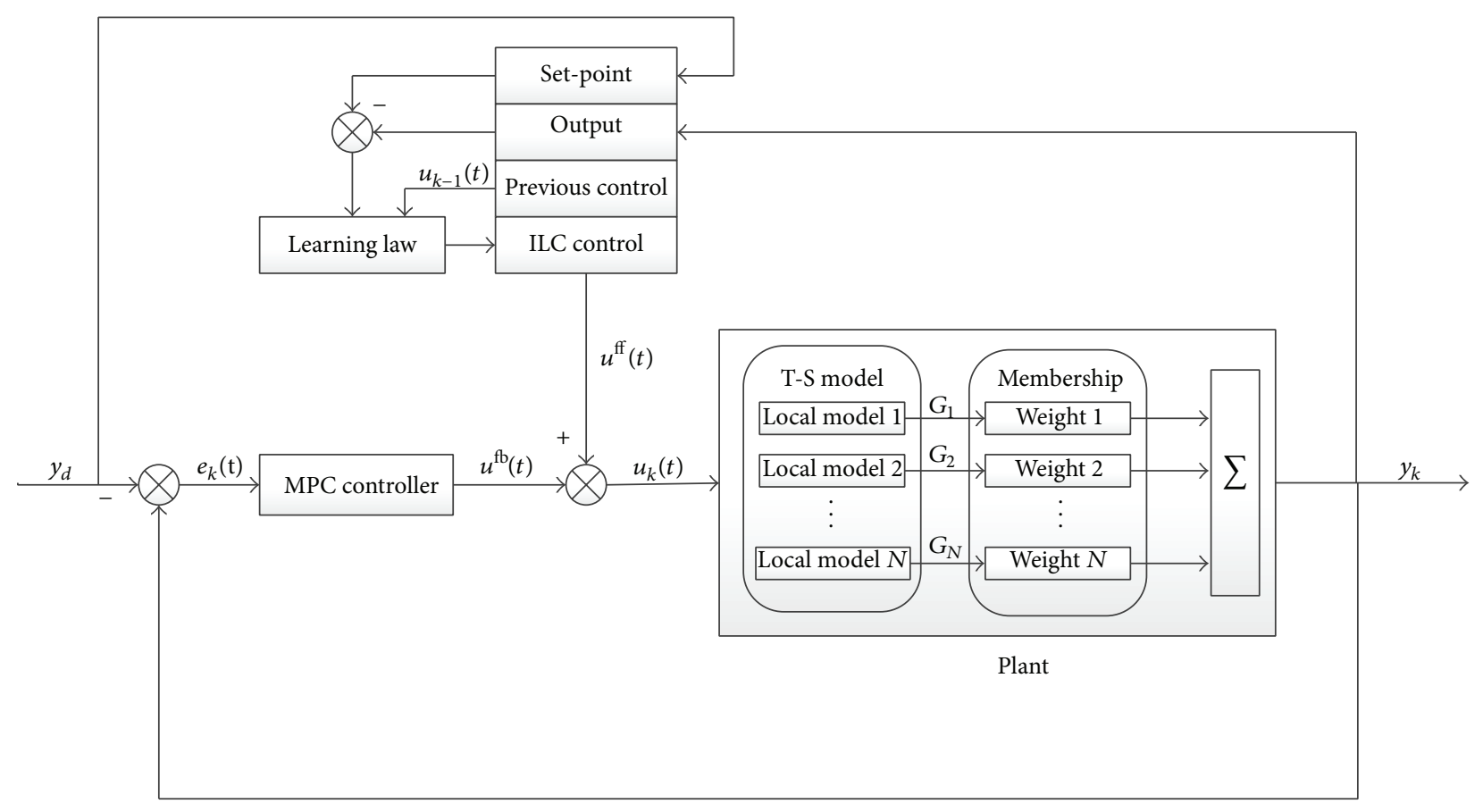

FIGURE 1: The control scheme for the feedback-assisted ILC controller.

where

$$
\begin{gathered}
z=\left(z_{1}, \ldots, z_{p}\right), \\
w_{i}(z)=\prod_{j=1}^{p} A_{i j}(z) .
\end{gathered}
$$

Let

$$
h_{i}(z)=\frac{w_{i}(z)}{\sum_{i=1}^{r} w_{i}(z)},
$$

where

$$
\begin{aligned}
h_{i}(z)= & \left(\begin{array}{cccc}
h_{i}(z(1)) & 0 & \cdots & 0 \\
0 & h_{i}(z(2)) & \cdots & 0 \\
& & \ddots & \\
0 & 0 & \cdots & h_{i}(z(N))
\end{array}\right) \\
& \in R^{n_{y} N \times n_{y} N} .
\end{aligned}
$$

According to (10)-(13),

$$
\bar{y}=\frac{\sum_{i=1}^{r} w_{i}(z) \bar{y}^{i}}{\sum_{i=1}^{r} w_{i}(z)}=\sum_{i=1}^{r} h_{i}(z) G^{i} u^{\mathrm{ff}}-\sum_{i=1}^{r} h_{i}(z) p^{i} .
$$

Define the weighted sum of disturbances and errors as

$$
D=\sum_{i=1}^{r} h_{i}(z) p^{i}
$$

Let $y_{d}$ represent the predefined output; the error trajectory $\bar{e}$ can be expressed as

$$
\bar{e}=y_{d}-\bar{y}=y_{d}-\sum_{i=1}^{r} h_{i}(z) G^{i} u^{\mathrm{ff}}+D .
$$

Generally, $D$ have both deterministic and stochastic components. In a repetitive system, it is convenient to express $D$ as the output of a linear stochastic system:

$$
\begin{gathered}
\bar{D}_{k+1}=\bar{D}_{k}+w_{k}, \\
D_{k}=\bar{D}_{k}+v_{k},
\end{gathered}
$$

where $k$ is the iteration number, $\bar{D}_{k}$ can be considered as the part of $D_{k}$ that will also appear in the next iteration run, and both $w_{k}$ and $v_{k}$ are zero-mean independent and identically distributed (i.i.d.) sequences in the $k$ th iteration with covariant matrices $R_{w}$ and $R_{v}$, respectively.

Define that $\tilde{e}$ is the part of $\bar{e}$ that will also appear in the next iteration. According to (16) and (17), we can formulate the expression of the tracking error sequences for two coherent iterations:

$$
\begin{aligned}
\bar{e}_{k} & =y_{d}-\sum_{i=1}^{r} h_{i}(z) G^{i} u_{k}^{\mathrm{ff}}+\bar{D}_{k}+v_{k}, \\
\widetilde{e}_{k} & =y_{d}-\sum_{i=1}^{r} h_{i}(z) G^{i} u_{k}^{\mathrm{ff}}+\bar{D}_{k}, \\
\bar{e}_{k+1} & =y_{d}-\sum_{i=1}^{r} h_{i}(z) G^{i} u_{k+1}^{\mathrm{ff}}+\bar{D}_{k+1}+v_{k+1}, \\
\widetilde{e}_{k+1} & =y_{d}-\sum_{i=1}^{r} h_{i}(z) G^{i} u_{k+1}^{\mathrm{ff}}+\bar{D}_{k+1} .
\end{aligned}
$$


Combining (17) with (18) can result in

$$
\begin{gathered}
\widetilde{e}_{k+1}=\widetilde{e}_{k}-\sum_{i=1}^{r} h_{i}(z) G^{i} \Delta u_{k+1}^{\mathrm{ff}}+w_{k}, \\
\bar{e}_{k}=\widetilde{e}_{k}+v_{k},
\end{gathered}
$$

where $\Delta u_{k+1}^{\mathrm{ff}}=u_{k+1}^{\mathrm{ff}}-u_{k}^{\mathrm{ff}}, \Delta$ represents a difference operator in terms of the iteration index.

When disturbances and noises in a specific iteration are significant, the following observer-based algorithm is adopted:

$$
u_{k}^{\mathrm{ff}}=u_{k-1}^{\mathrm{ff}}+\Delta u_{k}^{\mathrm{ff}}
$$

$\Delta u_{k}^{\mathrm{ff}}$ is determined by minimizing the following quadratic criterion:

$$
\min _{\Delta u_{k}^{f f}} \frac{1}{2}\left\{\left\|\tilde{e}_{k \mid k-1}^{T}\right\|_{S}^{2}+\left\|\Delta u_{k}^{\mathrm{ff}}\right\|_{T}^{2}\right\}
$$

According to (19), (20) is subject to

$$
\begin{gathered}
\tilde{e}_{k \mid k-1}=\widetilde{e}_{k-1 \mid k-1}-\sum_{i=1}^{r} h_{i}(z) G^{i} \Delta u_{k}^{\mathrm{ff}}, \\
\widetilde{e}_{k-1 \mid k-1}=\widetilde{e}_{k-1 \mid k-2}+K\left(\bar{e}_{k-1}-\widetilde{e}_{k-1 \mid k-2}\right),
\end{gathered}
$$

where $\widetilde{e}_{n \mid m}$ is an estimation of $\widetilde{e}_{n}$ that can be interpreted as using the information of $\widetilde{e}$ in the $m$ th iteration to estimate the behavior of $\widetilde{e}$ in the $n$th iteration; $S$ and Tare positive-definite matrices; $K$ is the Kalman gain.

The solution to the above is

$$
\begin{aligned}
\Delta u_{k}^{\mathrm{ff}}= & {\left[\left(\sum_{i=1}^{r} h_{i}(z) G^{i}\right)^{T} S\left(\sum_{i=1}^{r} h_{i}(z) G^{i}\right)+T\right]^{-1} } \\
& \times\left(\sum_{i=1}^{r} h_{i}(z) G^{i}\right)^{T} S \widetilde{e}_{k-1 \mid k-1}
\end{aligned}
$$

Consequently, the feedforward control signal at time $t$ is

$$
u_{k}^{\mathrm{ff}}(t)=u_{k-1}^{\mathrm{ff}}(t)+\Delta u_{k}^{\mathrm{ff}}(t)=u_{k-1}^{\mathrm{ff}}(t)+L \widetilde{e}_{k-1 \mid k-1},
$$

where

$$
\begin{aligned}
L= & (\underbrace{0}_{n_{u} \times n_{u} t} \cdots \underbrace{1}_{n_{u} \times n_{u}} \cdots \underbrace{0}_{n_{u} \times(N-t-1) n_{u}}) \\
& \cdot\left[\left(\sum_{i=1}^{r} h_{i}(z) G^{i}\right)^{T} S\left(\sum_{i=1}^{r} h_{i}(z) G^{i}\right)+T\right]^{-1} \\
& \times\left(\sum_{i=1}^{r} h_{i}(z) G^{i}\right)^{T} S .
\end{aligned}
$$

\section{The Feedback-Assisted Control Law}

In order to effectively reject nonrepeating disturbances in the current run, it is necessary to combine MPC with the above ILC to deduce the overall FF-FB control law.

For convenience, the following notation is introduced:

$$
\lambda\left(\begin{array}{l}
t_{1} \\
t_{2}
\end{array}\right)=\left[\begin{array}{c}
\lambda\left(t_{1}\right) \\
\lambda\left(t_{1}+1\right) \\
\vdots \\
\lambda\left(t_{2}\right)
\end{array}\right] .
$$

The process model (8) is used to deduce the revised MPC feedback control law. At time $t$,

$$
\widehat{y}_{k}^{i}(t)=g_{t}^{i} \widehat{u}_{k}\left(\left.\right|_{t-1} ^{0}\right),
$$

where $g_{t}^{i}$ denotes nonzero elements in $t$ th row vector of $G^{i}$.

At time $t$ in MPC, we partitioned the control signal as two parts to predict the output at time $t+j(j=1, \ldots, m)$ :

$$
\widehat{y}_{k}^{i}(t+j \mid t)=g_{t+j}^{i 1} \widehat{u}_{k}\left(\left.\right|_{t-1} ^{0}\right)+g_{t+j}^{i 2} \widehat{u}_{k}\left(\left.\right|_{t+j-1} ^{t}\right),
$$

where

$$
\begin{gathered}
g_{t+j}^{i 1}=\left[\begin{array}{llll}
g_{t+j, 0}^{i} & g_{t+j, 1}^{i} & \cdots & g_{t+j, t-1}^{i}
\end{array}\right], \\
g_{t+j}^{i 2}=\left[\begin{array}{llll}
g_{t+j, t}^{i} & g_{t+j, t+1}^{i} & \cdots & g_{t+j, t+j-1}^{i}
\end{array}\right] .
\end{gathered}
$$

Considering the predictive horizon is $m$, (29) can be expanded as

$$
\widehat{y}_{k}^{i}\left(\left|\begin{array}{l}
t+1 \\
t+m
\end{array}\right| t\right)=G_{t}^{i 1} \widehat{u}_{k}\left(\left.\right|_{t-1} ^{0}\right)+G_{t}^{i 2} \widehat{u}_{k}\left(\mid \begin{array}{l}
t \\
t+m-1
\end{array}\right),
$$

where

$$
\begin{gathered}
G_{t}^{i 1}=\left[\begin{array}{cccc}
g_{t+1,0}^{i} & g_{t+1,1}^{i} & \cdots & g_{t+1, t-1}^{i} \\
g_{t+2,0}^{i} & g_{t+2,1}^{i} & \cdots & g_{t+2, t-1}^{i} \\
\vdots & \vdots & \ddots & \vdots \\
g_{t+m, 0}^{i} & g_{t+m, 1}^{i} & \cdots & g_{t+m, t-1}^{i}
\end{array}\right], \\
G_{t}^{i 2}=\left[\begin{array}{cccc}
g_{t+1, t}^{i} & 0 & \cdots & 0 \\
g_{t+2, t}^{i} & g_{t+2, t+1}^{i} & \cdots & 0 \\
\vdots & \vdots & \ddots & \vdots \\
g_{t+m, t}^{i} & g_{t+m, t+1}^{i} & \cdots & g_{t+m, t+m-1}^{i}
\end{array}\right] .
\end{gathered}
$$

Similar to (14), the integral system output $\widehat{y}_{k}\left(\left.\right|_{t+m} ^{t+1} \mid t\right)$ can be expressed as

$$
\widehat{y}_{k}\left(\left|\begin{array}{l}
t+1 \\
t+m
\end{array}\right| t\right)=\sum_{i=1}^{r} H_{t}^{i} \widehat{y}_{k}^{i}\left(\left|\begin{array}{l}
t+1 \\
t+m
\end{array}\right| t\right),
$$

where

$$
\begin{aligned}
H_{t}^{i}= & \left(\begin{array}{cccc}
h_{i}(z(t+1)) & \cdots & 0 & 0 \\
0 & h_{i}(z(t+2)) & \cdots & 0 \\
& \ddots & & \\
0 & 0 & \cdots & h_{i}(z(t+m))
\end{array}\right) \\
& \in R^{n_{y} m \times n_{y} m} .
\end{aligned}
$$


The tracking error of the predictive model is defined as

$$
\begin{aligned}
\widehat{e}_{k}\left(\left|\begin{array}{l}
t+1 \\
t+m
\end{array}\right| t\right) & =y_{d}\left(\begin{array}{l}
t+1 \\
t+m
\end{array}\right)-\widehat{y}_{k}\left(\left|\begin{array}{l}
t+1 \\
t+m
\end{array}\right| t\right) \\
& =f_{t}-\sum_{i=1}^{r} H_{t}^{i} G_{t}^{i 2} \widehat{u}_{k}\left(\begin{array}{l}
t \\
t+m-1
\end{array}\right),
\end{aligned}
$$

where

$$
f_{t}=y_{d}\left(\mid \begin{array}{l}
t+1 \\
t+m
\end{array}\right)-\sum_{i=1}^{r} H_{t}^{i} G_{t}^{i 1} \widehat{u}_{k}^{i}\left(\left.\right|_{t-1} ^{0}\right) .
$$

Define the cost function of MPC as follows:

$$
\begin{aligned}
& \min _{\widehat{u}_{k}\left(\left.\right|_{t+m-1} ^{t}\right.} J_{k}(t) \\
& \quad=\frac{1}{2}\left\{\left\|\widehat{e}_{k}\left(\left|\begin{array}{l}
t+1 \\
t+m
\end{array}\right| t\right)\right\|_{Q}^{2}+\left\|\widehat{u}_{k}\left(\mid \begin{array}{l}
t \\
t+m-1
\end{array}\right)\right\|_{R}^{2}\right\},
\end{aligned}
$$

where $Q$ and $R$ are positive-definite matrices with appropriate dimensions.

According to (36), the feedback control signal can be obtained:

$$
\begin{aligned}
\widehat{u}_{k}\left(\left.\right|_{t+m-1} ^{t}\right)= & \left(\left(\sum_{i=1}^{r} H_{t}^{i} G_{t}^{i 2}\right)^{T} Q\left(\sum_{i=1}^{r} H_{t}^{i} G_{t}^{i 2}\right)+R\right)^{-1} \\
& \times\left(\sum_{i=1}^{r} H_{t}^{i} G_{t}^{i 2}\right)^{T} Q f_{t} .
\end{aligned}
$$

Only the first element $\widehat{u}_{k}(t)$ from $\widehat{u}_{k}\left(\left.\right|_{t+m-1} ^{t}\right)$ is used in the process at the current time $t$ as the feedback control signal.

According to (24) and (37), the overall control law is obtained as

$$
\begin{aligned}
u_{k}(t) & =u_{k}^{\mathrm{ff}}(t)+u_{k}^{\mathrm{fb}}(t) \\
& =u_{k-1}^{\mathrm{ff}}(t)+L \widetilde{e}_{k-1 \mid k-1}+\widehat{u}_{k}(t) .
\end{aligned}
$$

In industrial applications, various constraints are imposed on the input magnitude and the input change rate (in terms of time index), which are given as the following linear inequalities:

$$
\begin{gathered}
u^{\min } \leq u_{k}(t) \leq u^{\max }, \\
\delta u^{\min } \leq \delta u_{k}(t) \leq \delta u^{\max },
\end{gathered}
$$

where $\delta u_{k}(t)=u_{k}(t)-u_{k}(t-1), \delta$ represents a difference operator in terms of the time index and $u^{\text {min }}$ and $\delta u^{\text {min }}$ represent the lower limits of $u_{k}(t)$ and $\delta u_{k}(t)$, respectively; $u^{\max }$ and $\delta u^{\max }$ represent the upper limits of $u_{k}(t)$ and $\delta u_{k}(t)$, respectively.

In the FF-FB control scheme, the ILC feedforward signal $u_{k}^{\mathrm{ff}}$ is known before the $k$ th iteration.

Define

$$
\begin{aligned}
U^{\min } & =\left[\begin{array}{lll}
u^{\min T} & \cdots & u^{\min T}
\end{array}\right]^{T} \in R^{n_{u} m}, \\
U^{\max } & =\left[\begin{array}{lll}
u^{\max T} & \cdots & u^{\max T}
\end{array}\right]^{T} \in R^{n_{u} m} .
\end{aligned}
$$

Through some straightforward manipulation, the constraints imposed on $\widehat{u}_{k}\left(\left.\right|_{t+m-1} ^{t}\right)$ and $\delta \widehat{u}_{k}\left(\left.\right|_{t+m-1} ^{t}\right)$ can be expressed as

$$
\begin{aligned}
U^{\mathrm{min}}-u_{k}^{\mathrm{ff}}\left(\left.\right|_{t+m-1} ^{t}\right) & \leq \widehat{u}_{k}\left(\begin{array}{l}
t \\
t+m-1
\end{array}\right) \\
& \leq U^{\mathrm{max}}-u_{k}^{\mathrm{ff}}\left(\left.\right|_{t+m-1} ^{t}\right), \\
\delta U^{\mathrm{min}}-\delta u_{k}^{\mathrm{ff}}\left(\left.\right|_{t+m-1} ^{t}\right)+C_{2} & \leq C_{1} \widehat{u}_{k}\left(\left.\right|_{t+m-1} ^{t}\right) \\
& \leq \delta U^{\mathrm{max}}-\delta u_{k}^{\mathrm{ff}}\left(\left.\right|_{t+m-1} ^{t}\right)+C_{2},
\end{aligned}
$$

where

$$
\begin{aligned}
C_{1} & =\left(\begin{array}{ccccc}
I & I & \cdots & I & I \\
0 & I & \cdots & I & I \\
& & \ddots & & \\
I & I & \cdots & 0 & I
\end{array}\right) \in R^{n_{u} m \times n_{u} m} \\
C_{2} & =\left(\begin{array}{c}
\widehat{u}_{k}(t-1) \\
0 \\
\vdots \\
0
\end{array}\right) \in R^{n_{u} m}
\end{aligned}
$$

The constraints can be put in the general form of

$$
M \widehat{u}_{k}\left(\left.\right|_{t+m-1} ^{t}\right) \leq \Re
$$

where

$$
\begin{aligned}
& M=\left(\begin{array}{c}
I \\
-I \\
C_{1} \\
-C_{1}
\end{array}\right) \\
& \Re=\left(\begin{array}{c}
U^{\mathrm{max}}-u_{k}^{\mathrm{ff}}\left(\left.\right|_{t+m-1} ^{t}\right) \\
-U^{\mathrm{min}}+u_{k}^{\mathrm{ff}}\left(\left.\right|_{t+m-1} ^{t}\right) \\
\delta U^{\mathrm{max}}-\delta u_{k}^{\mathrm{ff}}\left(\left.\right|_{t+m-1} ^{t}\right)+C_{2} \\
-\delta U^{\mathrm{min}}+\delta u_{k}^{\mathrm{ff}}\left(\left.\right|_{t+m-1} ^{t}\right)-C_{2}
\end{array}\right) .
\end{aligned}
$$

In conditional MPC method, the constraints imposed on the practical system are often solved by a quadratic program (QP). The standard QP problem can be expressed as follows:

$$
\begin{aligned}
& \min _{\theta} J=\frac{1}{2} \theta^{T} \Phi \theta+\theta^{T} \phi, \\
& \text { s.t. } M \theta \leq \mathfrak{R},
\end{aligned}
$$

where (45) denotes the objective function and (46) denotes the constraints, the decision variable is $\theta, \Phi, \phi, M$, and $\Re$ are compatible matrices and vectors, and $\Phi$ is supposed to be a symmetric and positive definite matrix. 

tuted:

Combining (34), (36), and (43), a QP problem is consti-

$$
\begin{aligned}
\min _{\widehat{u}_{k}\left(\left.\right|_{t+m-1} ^{t}\right)} \bar{J}_{k}(t)= & \frac{1}{2} \widehat{u}_{k}^{T}\left(\left.\right|_{t+m-1} ^{t}\right) \Phi \widehat{u}_{k}\left(\left.\right|_{t+m-1} ^{t}\right) \\
& +\widehat{u}_{k}^{T}\left(\left.\right|_{t+m-1} ^{t}\right) \phi, \\
\text { s.t. } M \widehat{u}_{k}\left(\left.\right|_{t+m-1} ^{t}\right) & \leq \Re,
\end{aligned}
$$

where $\Phi=\left(\sum_{i=1}^{r} H_{t}^{i} G_{t}^{i 2}\right)^{T} Q\left(\sum_{i=1}^{r} H_{t}^{i} G_{t}^{i 2}\right)+R$,

$$
\phi=-\left(\sum_{i=1}^{r} H_{t}^{i} G_{t}^{i 2}\right)^{T} Q f_{t} .
$$

The QP problem is often solved by active set method and interior point method. In this case, the QP is solved in MATLAB by the function named quadprog for convenience. The first element which served as the optimal feedback control signal is applied to the process at the current time.

\section{Case Study}

The proposed feedback-assisted ILC has been simulated on a 160 MW drum-type boiler-turbine-generator plant represented by a third order MIMO coupling nonlinear model [13]:

$$
\begin{gathered}
\dot{x}_{1}=-0.0018 u_{2} x_{1}^{9 / 8}+0.9 u_{1}-0.15 u_{3}, \\
\dot{x}_{2}=\left(0.073 u_{2}-0.016\right) x_{1}^{9 / 8}-0.1 x_{2}, \\
\dot{x}_{3}=\frac{\left[141 u_{3}-\left(1.1 u_{2}-0.19\right) x_{1}\right]}{85}, \\
y_{1}=x_{1}, \\
y_{2}=x_{2}, \\
y_{3}=0.05\left(0.13073 x_{3}+100 \alpha_{\mathrm{cs}}+\frac{q_{e}}{9}-67.975\right), \\
\alpha_{\mathrm{cs}}=\frac{\left(1-0.001538 x_{3}\right)\left(0.8 x_{1}-25.6\right)}{x_{3}\left(1.0394-0.0012304 x_{1}\right)}, \\
q_{e}=\left(0.845 u_{2}-0.147\right) x_{1}+45.59 u_{1}-2.514 u_{3}-2.096,
\end{gathered}
$$

where $x_{1}, x_{2}$, and $x_{3}$ are drum steam pressure $\left(\mathrm{kg} / \mathrm{cm}^{2}\right)$, electric power (WM), and steam-water fluid density in the drum, respectively, the output $y_{3}$ is the drum water level $(\mathrm{cm})$ obtained using two algebraic calculations $\alpha_{\mathrm{cs}}$ and $q_{e}$ which are the steam quality and the evaporation rate $(\mathrm{kg} / \mathrm{s})$, and the inputs $u_{1}, u_{2}$, and $u_{3}$ are normalized positions of valve actuators that control the mass flow rates of fuel, steam to the turbine, and feedwater to the drum, respectively. The sampling period is 1 second. The constraints imposed on input change rates are

$$
\begin{gathered}
-0.007 \leq \delta u_{1}(t) \leq 0.007 \\
-2.0 \leq \delta u_{2}(t) \leq 0.02 \\
-0.05 \leq \delta u_{3}(t) \leq 0.05
\end{gathered}
$$

Though the model shows strong nonlinearity, one major characteristic of the boiler-turbine-generator is that the plant dynamic changes with load within the whole operating range. This property motivates many researchers to establish the fuzzy model by incorporating the human operators' experience [14]. Choose the operating points to be $(120,40,0)$, $(135,90,0.5)$, and $(150,140,1)$, which can be defined as low (L), medium (M), and high $(\mathrm{H})$, respectively. This division also uses the experience of the operators, who regard a load of $40 \mathrm{MW}$ as low, $90 \mathrm{MW}$ as medium, and $140 \mathrm{MW}$ as high. Therefore the rules can be constructed as follows:

$$
\begin{aligned}
& R_{1}^{1}: y_{1}^{1}(t)=108+0.9792 y_{1}(t-1)+9.5039 u_{1}(t-1) \\
& -298.1253 u_{2}(t-1)+115.7860 u_{3}(t-1) \text {, } \\
& R_{1}^{2}: y_{1}^{2}(t)=4.1736+0.9682 y_{1}(t-1)+8.9645 u_{1}(t-1) \\
& -4.2498 u_{2}(t-1)-1.5497 u_{3}(t-1) \text {, } \\
& R_{1}^{3}: y_{1}^{3}(t)=4.8814+0.9688 y_{1}(t-1)+8.9645 u_{1}(t-1) \\
& -5.2957 u_{2}(t-1)-1.4789 u_{3}(t-1) \text {, } \\
& R_{2}^{1}: y_{2}^{1}(t)=-33.7364+0.1868 y_{1}(t-1) \\
& +0.0491 y_{2}(t-1)+104.9749 u_{2}(t-1) \text {, } \\
& R_{2}^{2}: y_{2}^{2}(t)=-120.6358+0.7299 y_{1}(t-1) \\
& -0.3592 y_{2}(t-1)+209.4179 u_{2}(t-1) \text {, } \\
& R_{2}^{1}: y_{2}^{1}(t)=-170.5567+0.8565 y_{1}(t-1) \\
& +0.0195 y_{2}(t-1)+201.3431 u_{2}(t-1) \text {, } \\
& R_{3}^{1}: y_{3}^{1}(t)=-0.3727+0.2428 y_{3}(t-1)-0.8475 u_{1}(t-1) \\
& +0.7170 u_{2}(t-1)+0.8352 u_{3}(t-1) \text {, } \\
& R_{3}^{2}: y_{3}^{2}(t)=0.2704+0.1171 y_{3}(t-1)-0.1904 u_{1}(t-1) \\
& +0.1894 u_{2}(t-1)+0.0370 u_{3}(t-1) \text {, } \\
& R_{3}^{3}: y_{3}^{3}(t)=-0.0650+0.3692 y_{3}(t-1)-0.2430 u_{1}(t-1) \\
& +0.5564 u_{2}(t-1)-0.2019 u_{3}(t-1) \text {, }
\end{aligned}
$$

where the fuzzy sets are illustrated in Figures 2, 3, and 4.

The sampling step $N=1200$. The system is initially steady in the following states $X=(100,50,449.5), Y=(100,50,0)$, and $U=(0.271,0.604,0.336)$.

The turning parameters are as follows: the covariance matrices of stochastic disturbances are $R_{w}=R_{v}=0.05 \mathrm{I}$; the control and predictive horizons are $m=p=20 ; S, T$, $Q$, and $R$ are chosen as $0.1 I, 0.8 I, I$, and $0.025 I$, respectively; $U_{0}=(0.271,0.604,0.336)$ is the input in the 1st iteration.

Simulations are first made under some fixed value of electric load demands to demonstrate the learning ability of 


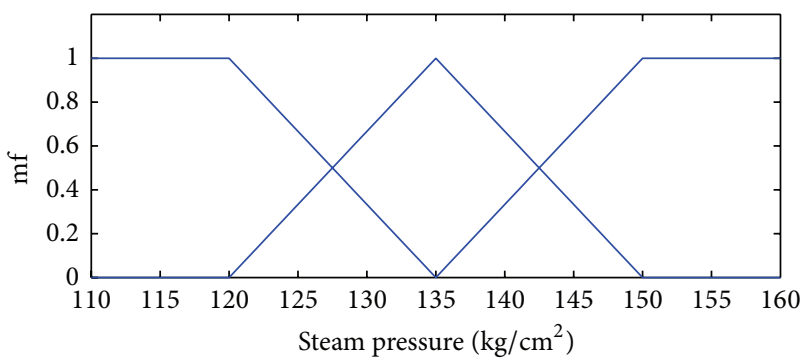

FIgURE 2: The fuzzy set of steam pressure.

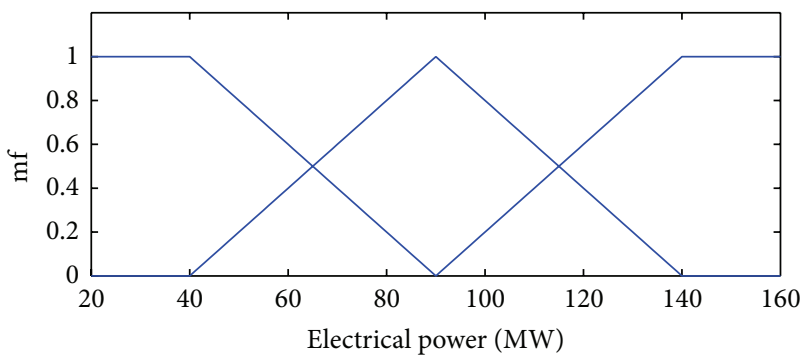

FIgURE 3: The fuzzy set of electric power.

the proposed feedback-assisted ILC. The references of steam pressure, electric power, and drum water level are

$$
\begin{aligned}
& y_{1}=110, \quad y_{2}=80, \quad y_{3}=0, \quad 0 \leq t \leq 400, \\
& y_{1}=120, \quad y_{2}=100, \quad y_{3}=0, \quad 40 \leq t \leq 800, \\
& y_{1}=130, \quad y_{2}=120, \quad y_{3}=0, \quad 800 \leq t \leq 1200 .
\end{aligned}
$$

Figures 5, 6, and 7 show the simulation results.

Figures 5-7 represent the outputs in the 1st, 4 th, 8 th, 10th, 15th, and 20th iteration, respectively. In order to make a comparison, the reference trajectories are also included in each group curve. It is obvious that the outputs of steam pressure, electric power, and drum water level converge to their desired references perfectly after several iterations. With the increase of the iteration run, the tracking property of the proposed strategy is improved. It shows that the algorithm can be applied for power plant control to attain wide-range operation.

The comparing results between the proposed feedbackassisted ILC and the pure ILC are shown in Figures 8, 9, 10, 11,12 , and 13 for the 8th iteration, under the case $y_{1}=120$, $y_{2}=100, y_{3}=0$. It can be seen that the proposed feedbackassisted ILC achieves a better tracking property over pure ILC for agreeing with the predefined steam pressure, electric power, and drum water level. Figures 11-13 show the total control signal $u_{i}$, feedforward signal $u_{i}^{\mathrm{ff}}$, and feedback signal $u_{i}^{\mathrm{fb}}$ of the three input signals, respectively. The actual input signal is in accordance with the expected behaviour. The feedforward signal provides most of control signals and the feedback control signal compensates for uncertainties and disturbances.

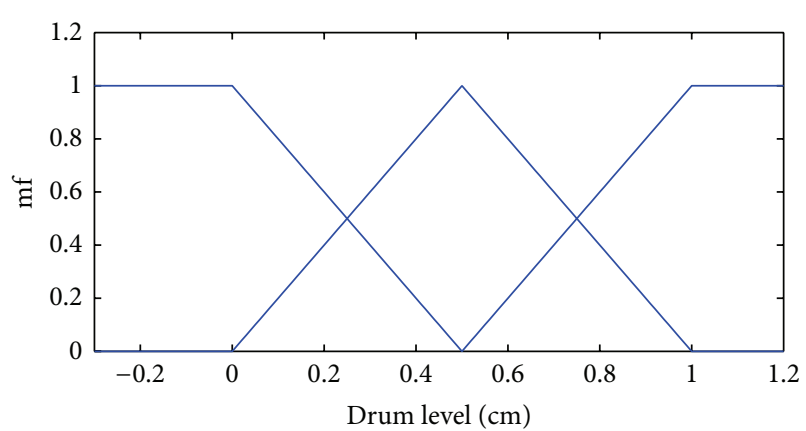

Figure 4: The fuzzy set of drum level.

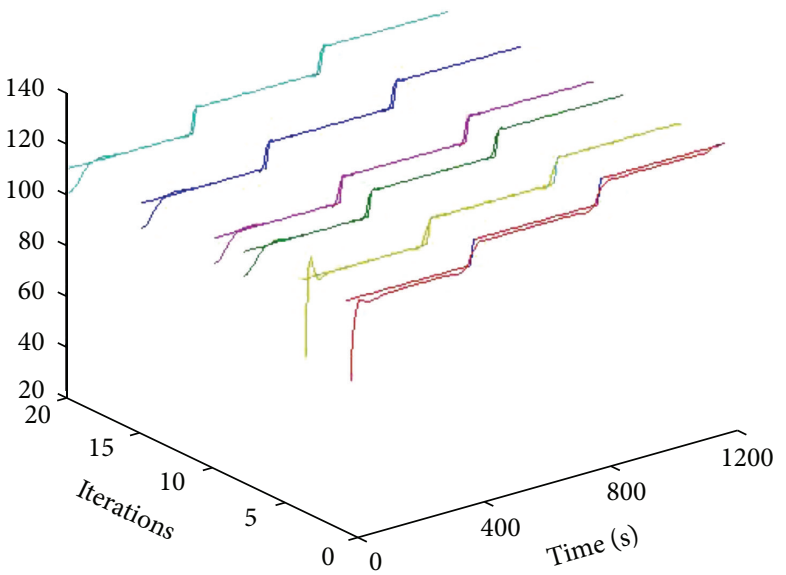

FIgURE 5: The steam pressure $\left(\mathrm{kg} / \mathrm{cm}^{2}\right)$.

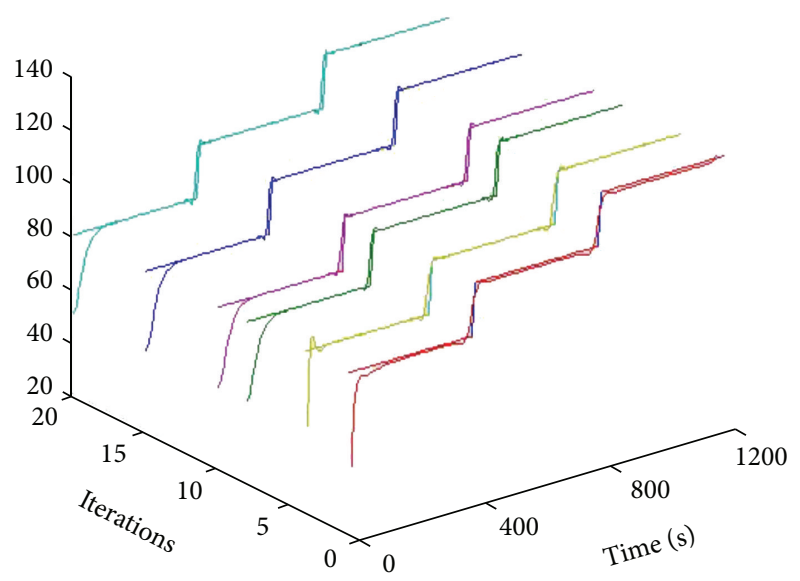

FIGURE 6: The electric power (MW).

In order to make a total comparison, define the sum of squared errors (SSE) between the reference trajectory and the actual output:

$$
\operatorname{SSE}(k)=\sum_{t=1}^{N}\left(y_{d}(t)-y_{k}(t)\right)^{2}, \quad k \in[1,20] .
$$




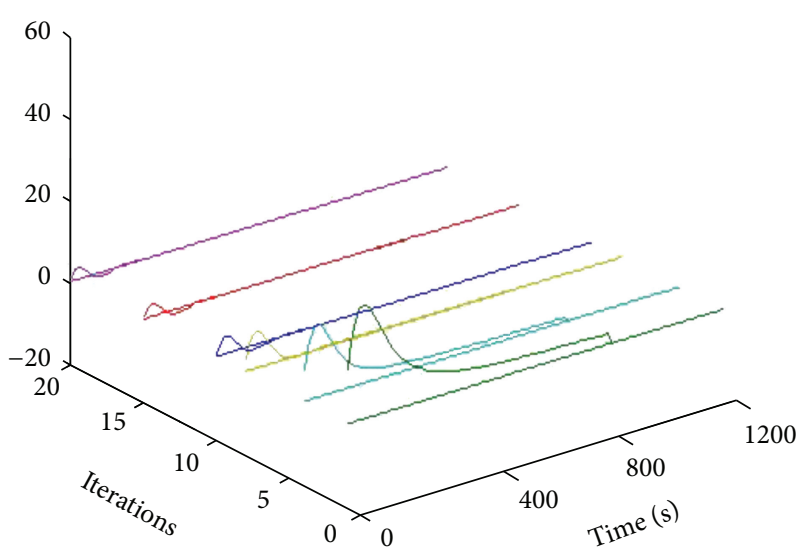

Figure 7: The drum water level (cm).

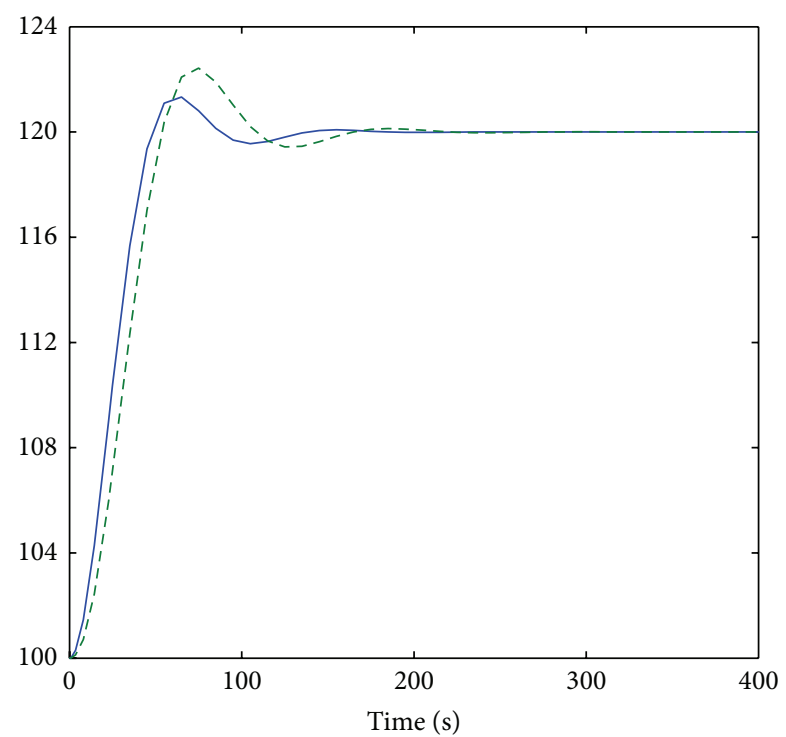

- Feedback-assisted ILC

- - ILC

Figure 8: The steam pressure $\left(\mathrm{kg} / \mathrm{cm}^{2}\right)$.

The qualitative difference is analyzed under the case $y_{1}=120$. Comparison is then made between the proposed feedback-assisted ILC and the pure feedback MPC. The feedback MPC adopts the same algorithm as depicted above. Figure 14 shows the comparing result of the tracking performance with respect to the iterations. It is clearly seen that although the proposed control scheme has a larger SSE in the initial iterations than the MPC, it converges to a smaller SSE boundary after the 5 th iteration. Meanwhile, the feedback MPC almost has the same tracking performance for the system, due to the fact that it cannot learn from the previous runs. The repetitive errors exist from the 1st iteration and its tracking property cannot be improved in the iteration process [15]. In this case, because of the plant model mismatch, the controller leaves repetitive tracking errors so the feedback MPC loses its efficacy for this kind of system.

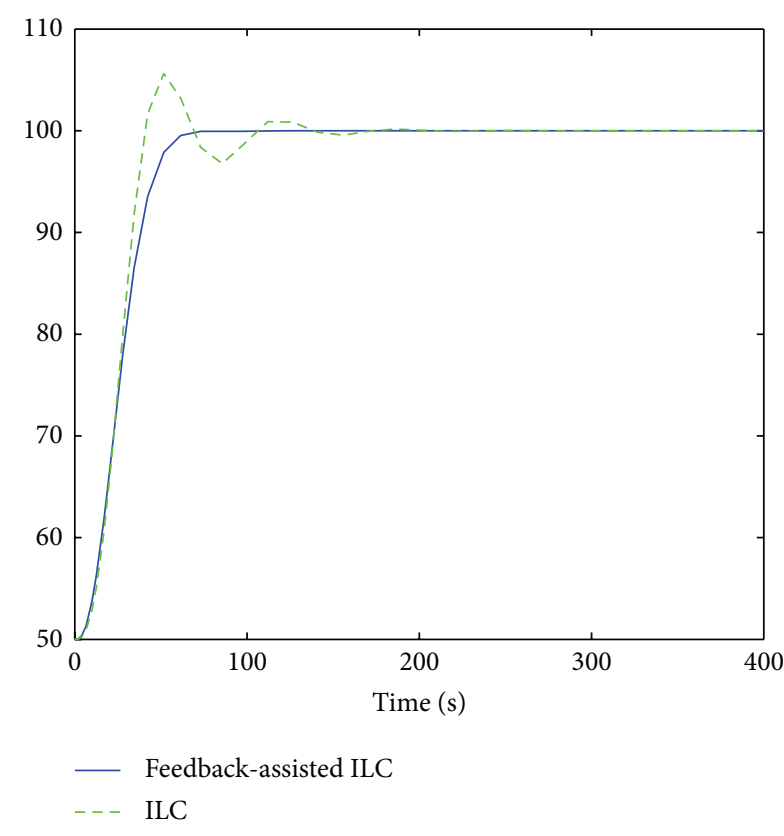

FIgURE 9: The electric power (MW).

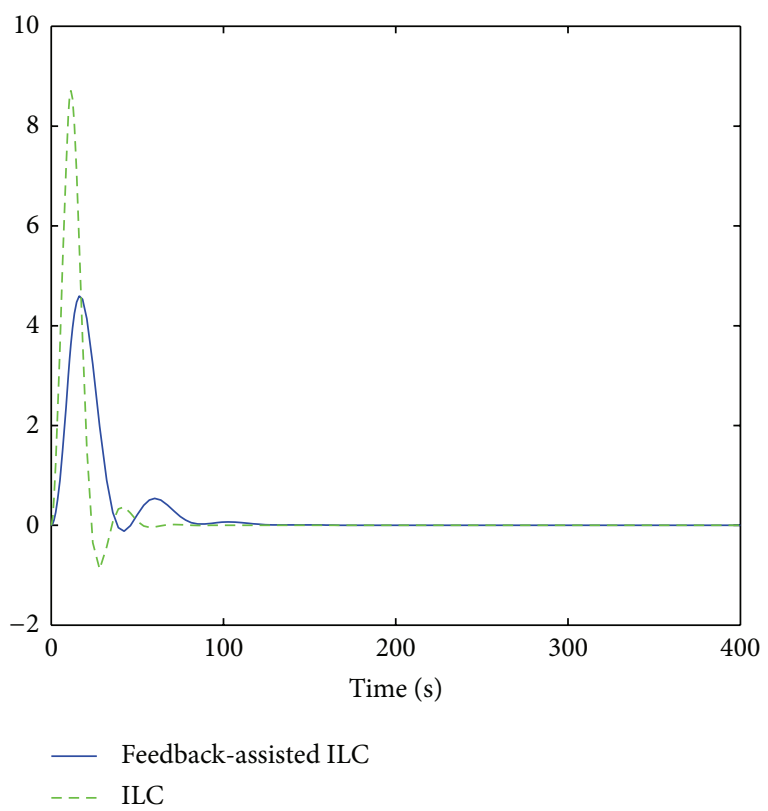

FIgURe 10: The drum water level $(\mathrm{cm})$.

\section{Conclusion}

In this paper, a feedback-assisted iterative learning controller is derived to track the predefined trajectory for a steamboiler nonlinear system whose dynamic can be presented by a T-S fuzzy model. MPC is appropriately incorporated into ILC as a feedback controller to eliminate the realtime random disturbances. Because of the using T-S model, the proposed FF-FB control strategy can attain wide-range load-following operation. The feedforward ILC control signal drives the process close to the desired reference. Meanwhile, 


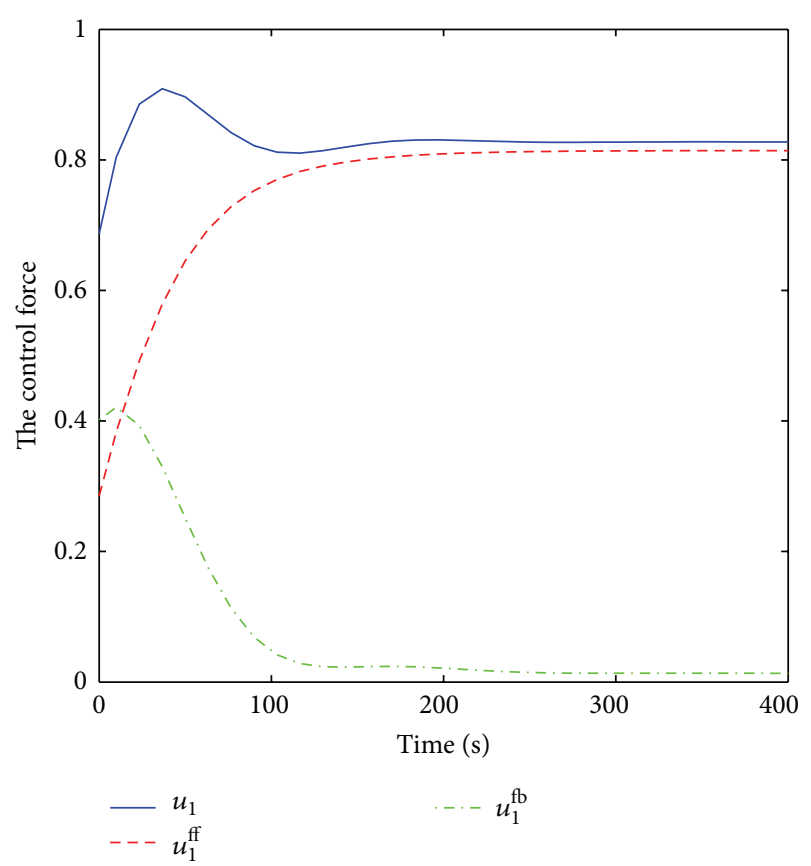

Figure 11: The control efforts of fuel valve.

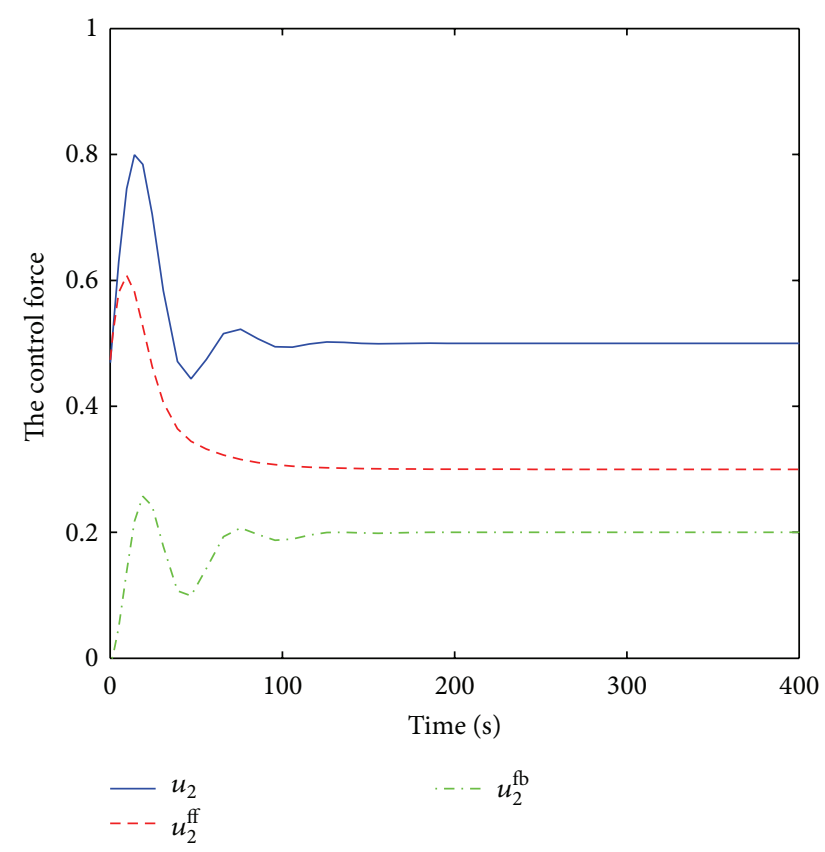

FIgURE 12: The control efforts of steam valve.

the feedback MPC control signal compensates for uncertainties and disturbances left by the ILC control signal. The simulation results demonstrate that the performance can be improved significantly under the proposed strategy than pure ILC and MPC.

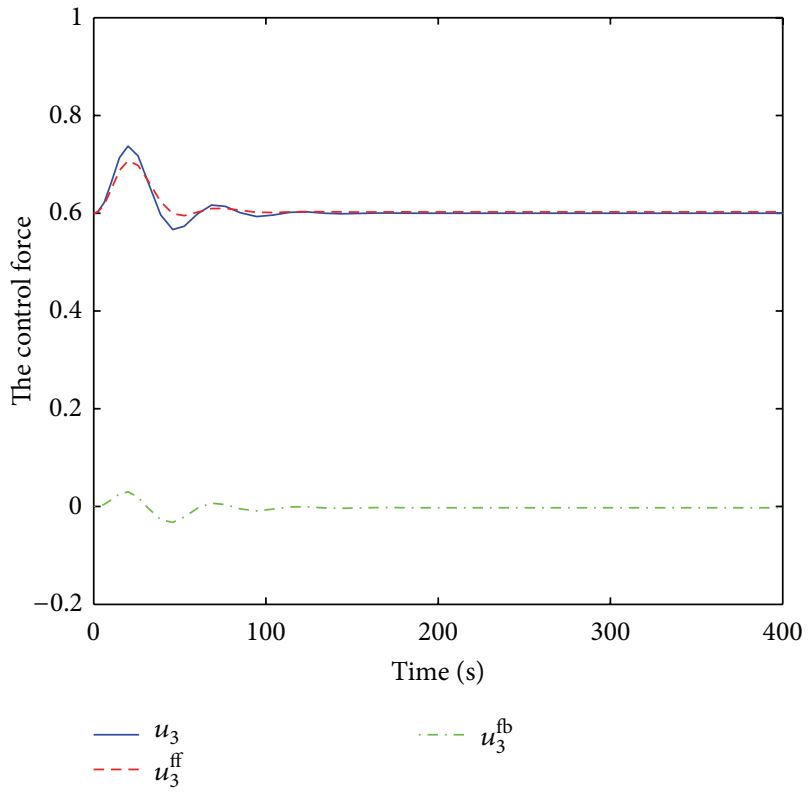

FIGURE 13: The control efforts of feedwater valve.

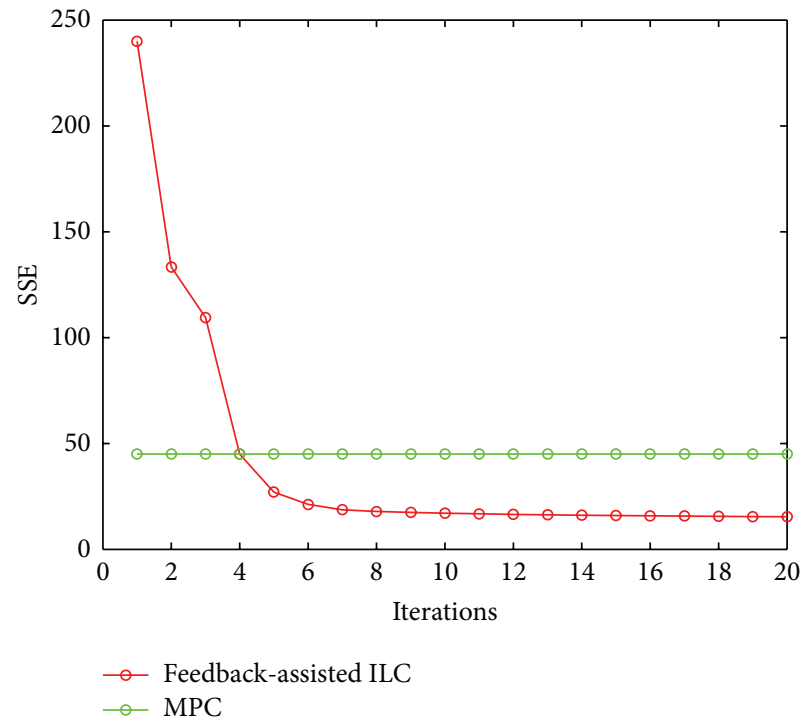

FIGURE 14: Comparison of tracking performance between the feedback-assisted ILC and feedback MPC.

\section{Conflict of Interests}

The authors declare that there is no conflict of interests regarding the publication of this paper.

\section{Acknowledgments}

This work was supported by National Natural Science Foundation of China under Grants 60974051 and 61273144 and by Natural Science Foundation of Beijing under Grant 4122071. 


\section{References}

[1] S. Arimoto, S. Kawamura, and F. Miyazaki, "Bettering operation of robots by learning," Journal of Robotic Systems, vol. 1, no. 2, pp. 123-140, 1984.

[2] M. Norrlof, "An adaptive iterative learning control algorithm with experiments on an industrial robot," IEEE Transactions on Robotics and Automation, vol. 18, no. 2, pp. 245-251, 2002.

[3] A. Tayebi, "Adaptive iterative learning control for robot manipulators," Automatica, vol. 40, no. 7, pp. 1195-1203, 2004.

[4] J. X. Xu, Y. Chen, T. H. Lee, and S. Yamamoto, “Terminal iterative learning control with an application to RTPCVD thickness control," Automatica, vol. 35, no. 9, pp. 1535-1542, 1999.

[5] D. H. Owens and J. Hatonen, "Iterative learning control—an optimization paradigm," Annual Reviews in Control, vol. 29, no. 1, pp. 57-70, 2005.

[6] K. L. Moore, Y. Q. Chen, and V. Bahl, "Monotonically convergent iterative learning control for linear discrete-time systems," Automatica, vol. 41, no. 9, pp. 1529-1537, 2005.

[7] J. X. Xu and Y. Tan, "Robust optimal design and convergence properties analysis of iterative learning control approaches," Automatica, vol. 38, no. 11, pp. 1867-1880, 2002.

[8] N. Amann, D. H. Owens, and E. Rogers, "Iterative learning control for discrete-time systems with exponential rate of convergence," IEE Proceedings-Control Theory and Applications, vol. 143, no. 2, pp. 217-224, 1996.

[9] K. S. Lee, J. H. Lee, I. S. Chin, and H. J. Lee, "Model predictive control technique combined with iterative learning for batch processes," AIChE Journal, vol. 45, no. 10, pp. 2175-2187, 1999.

[10] I. Chin, S. J. Qin, K. S. Lee, and M. Cho, "A two-stage iterative learning control technique combined with real-time feedback for independent disturbance rejection," Automatica, vol. 40, no. 11, pp. 1913-1922, 2004.

[11] T. Takagi and M. Sugeno, "Fuzzy identification of systems and its applications to modeling and control," IEEE Transactions on Systems, Man, and Cybernetics, vol. 15, no. 1, pp. 116-132, 1985.

[12] R. Garduno-Ramirez and M. Sanchez-Parra, "Control system modernization: turbogas unit case study," in Proceedings of the IFAC Symposium on Control of Power Plants and Power Systems (Sipower '95), vol. 2, pp. 245-250, Cancum, Mexico, December 1995.

[13] R. D. Bell and K. J. Astrom, "Dynamic models for boilerturbine-alternator units: data logs and parameter estimation for a 160 MW unit," Techincal Report TRFT-3192, Lund Institute of Technology, Lund, Sweden, 1987.

[14] Uk.-Y. Huh and J.-H. Kim, "MIMO fuzzy model for boilerturbine systems," in Proceedings of the 5th IEEE International Conference on Fuzzy Systems, vol. 1, pp. 541-547, September 1996.

[15] J. H. Lee, S. Natarajan, and K. S. Lee, "A model-based predictive control approach to repetitive control of continuous processes with periodic operations," Journal of Process Control, vol. 11, no. 2, pp. 195-207, 2001. 


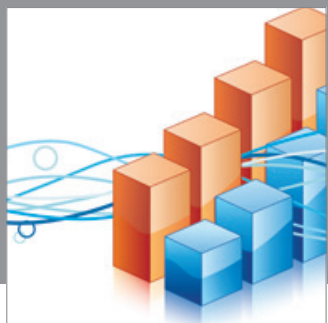

Advances in

Operations Research

mansans

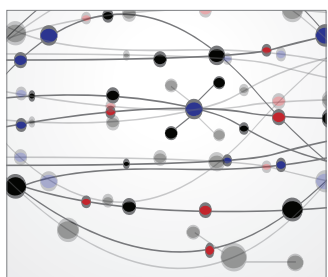

The Scientific World Journal
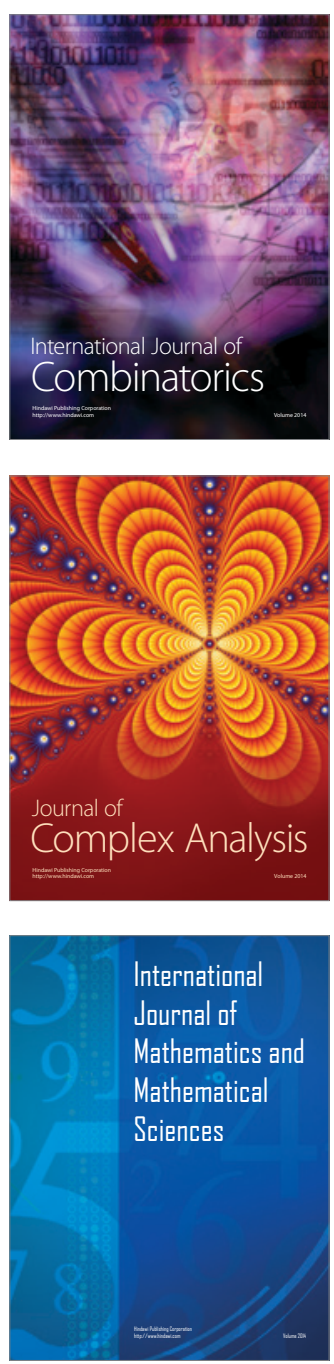
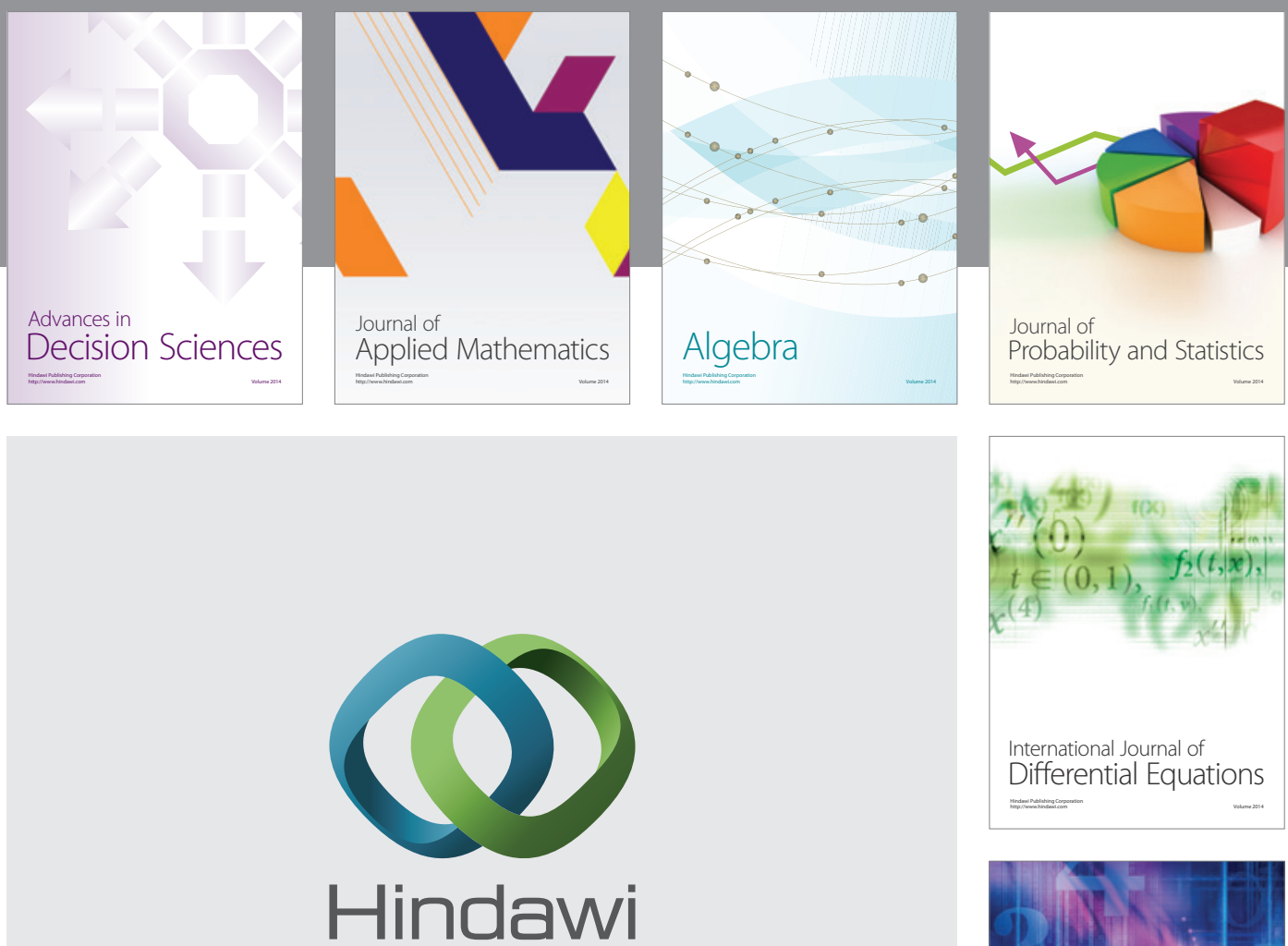

Submit your manuscripts at http://www.hindawi.com
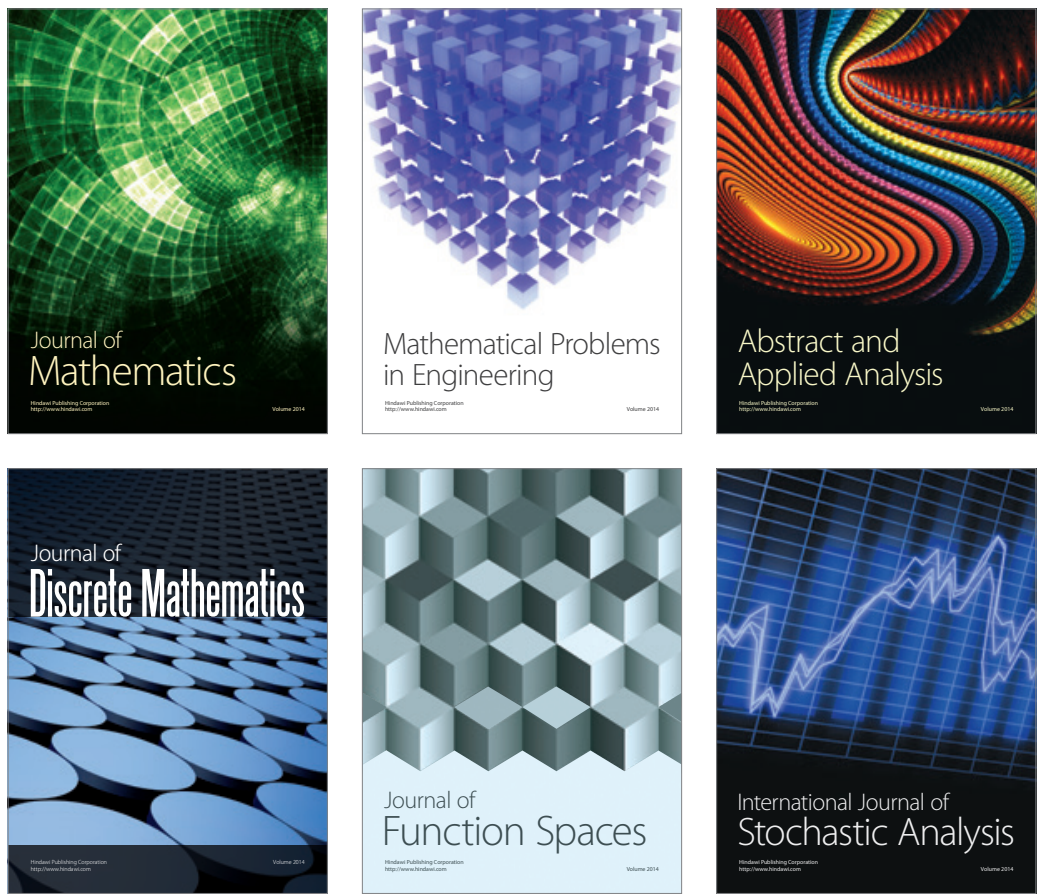

Journal of

Function Spaces

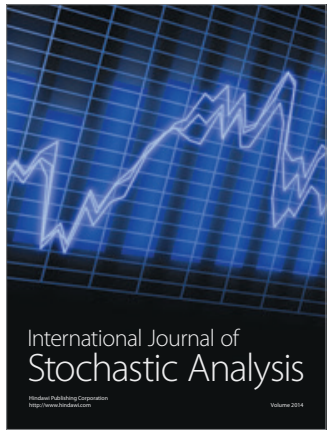

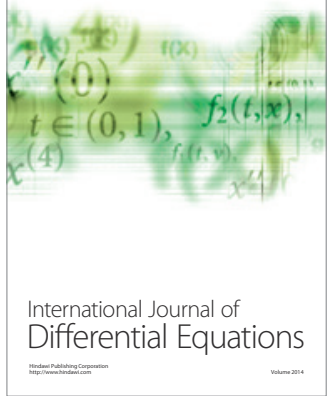
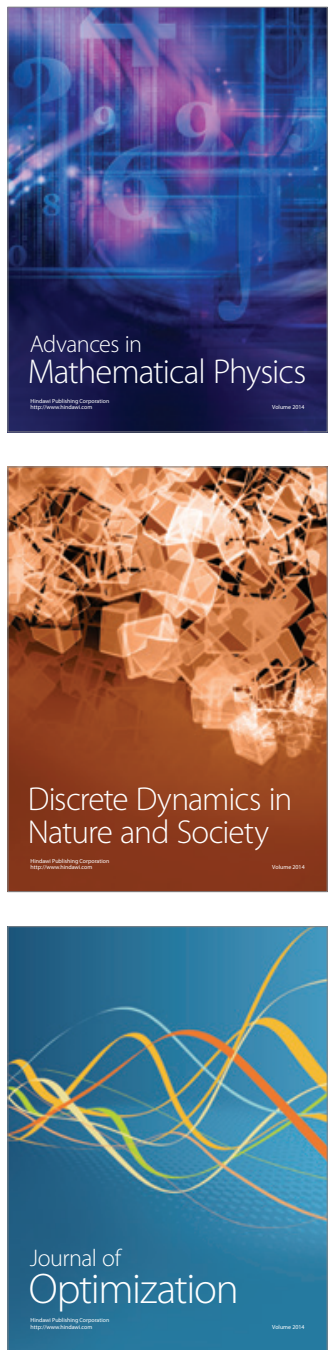